\author{
Ecología
}

\title{
Distribución y diversidad de dinoflagelados simbióticos en corales pétreos de la costa de Oaxaca, Pacífico de México
}

\section{Distribution and diversity of symbiotic dinoflagellates in stony corals off the coast of Oaxaca, Mexican Pacific}

\author{
Mariana Walther-Mendoza $^{\mathrm{a}, \mathrm{d}}$, Héctor Reyes-Bonilla ${ }^{\mathrm{a}, *}$, Todd C. LaJeunesse ${ }^{\mathrm{b}}$ \\ y Andrés López-Pérez ${ }^{\mathrm{c}}$ \\ ${ }^{a}$ Departamento Académico de Biología Marina, Universidad Autónoma de Baja California Sur, Carretera al sur, km. 5.5, \\ 23080, La Paz, Baja California Sur, México \\ ${ }^{\mathrm{b}}$ Department of Biology, Pennsylvania State University, University Park, Pensilvania, Estados Unidos de América \\ ${ }^{\mathrm{c}}$ Laboratorio Ecosistemas Costeros, Departamento Hidrobiología, Universidad Autónoma Metropolitana-Iztapalapa, San Rafael Atlixco 186, \\ Col. Vicentina, 09340, Ciudad de México, México \\ d Programa de Pesca Sustentable, Sociedad de Historia Natural Niparajá, A.C., Revolución de 1910, Núm. 430, 23020, La Paz, \\ Baja California Sur, México
}

Recibido el 5 de agosto de 2015; aceptado el 11 de noviembre de 2015

Disponible en Internet el 27 de mayo de 2016

\begin{abstract}
Resumen
Los corales arrecifales están asociados a dinoflagelados simbiontes —zooxantelas_ que proveen alimento a su hospedero. Para la costa occidental de México hay limitada información sobre este tipo de mutualismo y el objetivo del trabajo fue caracterizar las poblaciones de zooxantelas presentes en corales de la costa del estado de Oaxaca. Se recolectaron muestras de 3 géneros de coral: Pocillopora, Pavona y Porites, en 6 localidades y a distintas profundidades. Se extrajo ADN de las microalgas y se amplificó la región ITS2 para identificar los clados mediante electroforesis en gel de gradiente desnaturalizante. Se identificaron 7 tipos de zooxantelas pertenecientes al clado C y uno del D, evidenciando que en Oaxaca la diversidad es alta comparada con otras regiones del Pacífico mexicano. La mayor variedad de Symbiodinium se encontró en el género Porites (gonocórico), seguida de Pavona (hermafrodita) y, por último, Pocillopora (de frecuente reproducción asexual y en el cual todas las especies y colonias albergaron zooxantelas D1), lo que indica una relación entre la diversidad genética de las microalgas y el modo de reproducción coralino. Se observó aislamiento genético por distancia, composición variable de simbiontes por localidad y la presencia de clados privados; ello apunta hacia un flujo genético relativamente limitado de Symbiodinium en la región.

Derechos Reservados (C) 2016 Universidad Nacional Autónoma de México, Instituto de Biología. Este es un artículo de acceso abierto distribuido bajo los términos de la Licencia Creative Commons CC BY-NC-ND 4.0.
\end{abstract}

Palabras clave: Symbiodinium; Clados; ITS2; Holobionte; Reproducción del coral; Pocillopora; Pavona; Porites

\section{Abstract}

Reef corals are associated with symbiont dinoflagellates — zooxanthellae- which provide food to its host. There is limited information about this kind of mutualism in the western coast of Mexico, and thus the objective of this paper was to characterize the zooxanthellae populations maintained by corals at the coast of the state of Oaxaca. Samples of 3 coral genera were taken: Pocillopora, Pavona and Porites, at 6 locations and at different depths. Microalgal DNA was extracted, and the ITS2 region was amplified to identify clades using denaturing gradient gel electrophoresis. Seven zooxanthellae types were identified from clade $\mathrm{C}$, and a single one from clade $\mathrm{D}$, evidencing that in Oaxaca the diversity of zooxanthellae is high compared with other regions of the Mexican Pacific. The highest variety of Symbiodinium was found in the genus Porites (gonochoric) followed by Pavona (hermaphroditic), and finally in Pocillopora (of frequent asexual reproduction, and in which all species and colonies hosted type D1),

\footnotetext{
* Autor para correspondencia.

Correo electrónico: hreyes@uabcs.mx (H. Reyes-Bonilla).

La revisión por pares es responsabilidad de la Universidad Nacional Autónoma de México.
} 
which suggests a relationship between genetic diversity in the microalgae and the reproductive mode of the coral. Genetic isolation for distance was observed, as well as a variable composition of symbiont type at each sampling location, and the presence of private clades; these findings point towards a relatively limited gene flow of Symbiodinium in the region.

All Rights Reserved (C) 2016 Universidad Nacional Autónoma de México, Instituto de Biología. This is an open access item distributed under the Creative Commons CC License BY-NC-ND 4.0.

Keywords: Symbiodinium; Clades; ITS2; Holobiont; Coral reproduction; Pocillopora; Pavona; Porites

\section{Introducción}

Los arrecifes de coral se encuentran entre los ecosistemas más productivos y con mayor riqueza biológica del planeta. Juegan un papel ecológico y económico vital, ya que participan en los ciclos biogeoquímicos globales, proveen zonas de crianza para peces e invertebrados, dan protección a la línea de costa $\mathrm{y}$ abastecen a muchas poblaciones con bienes y servicios tales como alimento, posibilidades recreacionales y beneficios culturales (Done, Ogden, Wiebe y Rosen, 1996; Hoegh-Guldberg et al., 2007; Peterson y Lubchenco, 1997). Los corales que los forman se caracterizan por ser hipercalcificadores, ya que extraen cantidades masivas de carbonato de calcio del medio y lo depositan en forma de esqueleto y, para lograr esto, se valen de la relación simbiótica con dinoflagelados fotosintéticos llamados zooxantelas (Stanley, 2003). Estos organismos viven dentro del endodermo del coral en densidades altas (superiores a $10^{6}$ cél $/ \mathrm{cm}^{2}$ ), y proveen más del $95 \%$ de los requerimientos nutricionales a la colonia hospedera mediante la translocación intracelular de los productos resultantes de la fotosíntesis, como hidratos de carbono y lípidos (van Oppen, 2007). También, las microalgas facilitan la asimilación y conservación del nitrógeno, nutriente limitante en estos ambientes (Muller-Parker y D'Elia, 1997). Por su parte, el coral aporta a las zooxantelas sus productos de desecho como nutrientes inorgánicos del metabolismo incluyendo amonio y bióxido de carbono, y, además, provee un ambiente idóneo para la supervivencia del dinoflagelado (Sheppard, Davy y Pilling, 2009).

Para que se lleve a cabo la asociación entre la zooxantela y el coral se requieren condiciones ambientales específicas $\mathrm{y}$, por esto, la distribución del holobionte está limitada a la zona eufótica de los trópicos (Veron, 2000). La disrupción de estas asociaciones resulta en el fenómeno conocido como «blanqueamiento», que representa la pérdida parcial o total de algas simbióticas o pigmentos fotosintéticos, que resulta en un esqueleto de carbonato de calcio visible a través del tejido transparente (van Oppen y Lough, 2009). Los corales se blanquean en respuesta a una variedad de presiones ambientales como lo son variaciones drásticas en la temperatura, baja salinidad y contaminación, entre otras (Glynn, 1996).

En las últimas décadas se ha visto un incremento tanto en la frecuencia como en la intensidad de eventos de blanqueamiento de coral, considerándose una de las principales amenazas a los ecosistemas coralinos (van Oppen y Lough, 2009). Algunos efectos causados por esta manifestación son la disminución en las tasas de crecimiento coralino y calcificación, y la muerte del tejido del animal (Baker, Glynn y Riegl, 2008), factores que afectan a largo plazo a la comunidad, ya que causan pérdidas en la capacidad de las colonias para reproducirse y para competir contra otras especies. Como resultado, el arrecife se degrada, por lo que se vuelve menos productivo y disminuye, así, la calidad de sus servicios ambientales (Veron et al., 2009).

El fenómeno del blanqueamiento ha incrementado el interés en la biología de las zooxantelas en las últimas décadas. Existen múltiples estudios fisiológicos, ecológicos y filogenéticos de estos simbiontes, los cuales se han usado para ayudar en su identificación y clasificación. Debido a la morfología simple del dinoflagelado, se pensaba que había una sola especie cosmopolita llamada Symbiodinium microadriaticum Freudenthal, 1962. Sin embargo, ahora se sabe que el género es muy diverso (LaJeunesse, 2001). A nivel genético las zooxantelas se dividen en 9 «clados» designados de la «A» a la «I» (Baker, 2003; Fabina, Putnam, Franklin, Stat y Gates, 2012; Pochon, Montoya-Burgos, Stadelmann y Pawlowski, 2006; Rowan y Powers, 1991); cada uno se diferencia en sus intervalos de tolerancia a factores ambientales y habilidad para habitar diferentes hospederos, hábitats, profundidades e intensidades de luz (Pochon et al., 2006; Stanley, 2003). Por lo anterior, la fisiología del simbionte puede determinar en gran medida la susceptibilidad de algunos corales al estrés térmico, así como también brindar resistencia a eventos de blanqueamiento (Iglesias-Prieto y Trench, 1997; Rowan, Knowlton, Baker y Jara, 1997; Warner, Fitt y Schmidt, 1996).

La diversidad del género Symbiodinium y el grado de especificidad que existe con sus hospederos se conoce en diversos lugares del mundo (van Oppen y Lough, 2009). Sin embargo, en el Pacífico mexicano solamente se ha caracterizado el tipo de simbiontes de corales residentes en el sur de la península de Baja California y en la bahía de Banderas (Iglesias-Prieto, Beltrán, LaJeunesse, Reyes-Bonilla y Thomé, 2004; LaJeunesse, ReyesBonilla y Warner, 2007; LaJeunesse et al., 2008). Una de las áreas arrecifales más importantes en el Pacífico mexicano es la costa de Oaxaca, por presentar una de las más altas riquezas específicas y abundancias de coral dentro del Pacífico oriental tropical, así como por encontrarse junto al golfo de Tehuantepec, una importante zona de surgencias (López-Pérez et al., 2008; Reyes-Bonilla y López-Pérez, 1998). La región fue muy afectada por el fenómeno de oscilación sureña El Niño de 19971998, cuando se documentó una mortalidad coralina superior al 60\% (Reyes-Bonilla, Carriquiry, Leyte-Morales y CupulMagaña, 2002), pero afortunadamente la recuperación arrecifal ha sido excelente (López-Pérez y Hernández-Ballesteros, 2004). A partir de lo anterior, se consideró importante hacer un reconocimiento de la situación actual de los simbiontes que se encuentran en la zona, por lo que el objetivo del presente trabajo fue evaluar la composición y diversidad genética de los 
clados de Symbiodinium presentes en corales pétreos de diversas localidades arrecifales de la costa de Oaxaca, en el Pacífico sur mexicano, comparar su distribución en diferentes estratos de profundidad, y determinar si existe relación entre la distancia geográfica entre arrecifes y la distancia genética definida por las infracomunidades formadas por los clados.

\section{Materiales y métodos}

La región de estudio comprende la porción central del litoral del estado de Oaxaca; incluye 6 localidades: Mazunte, Ixtacahuite, La Mina, La Tijera, Bahía San Agustín y La Entrega, todas con presencia de arrecifes coralinos (fig. 1). El área de estudio presenta un clima cálido subhúmedo con lluvias en verano e invierno seco (Conanp, 2003; García, Vidal-Zepeda y Hernández, 1990). De octubre a marzo, el golfo de Tehuantepec es afectado por chorros de viento intensos en dirección norte-sur ( 15 a 20 m/s; Färber-Lorda, Lavín y Guerrero-Ruiz, 2004), llamados «tehuanos», que provocan procesos de surgencias (Pennington et al., 2006) y favorecen la mezcla de masas de agua y el transporte de nutrientes hacia la superficie.

Las principales corrientes marinas que afectan la costa de Oaxaca son la contracorriente norecuatorial y la corriente costera de Costa Rica (Kessler, 2006). La temperatura media del mar es de $28.2^{\circ} \mathrm{C}$ y el promedio de salinidad es de 34.5 UPS (Pennington et al., 2006). En la porción oceánica del golfo de Tehuantepec - fuera de la plataforma continental—, la concentración superficial de nutrientes tiene valores de $2.9 \mu \mathrm{mol} 1^{-1}$ para nitratos, $0.2 \mu \mathrm{mol}$ para fosfatos, $10.1 \mu \mathrm{mol}$ para silicatos y $0.33 \mathrm{mg} \mathrm{m}^{-3}$ para clorofila (Pennington et al., 2006). El régi-
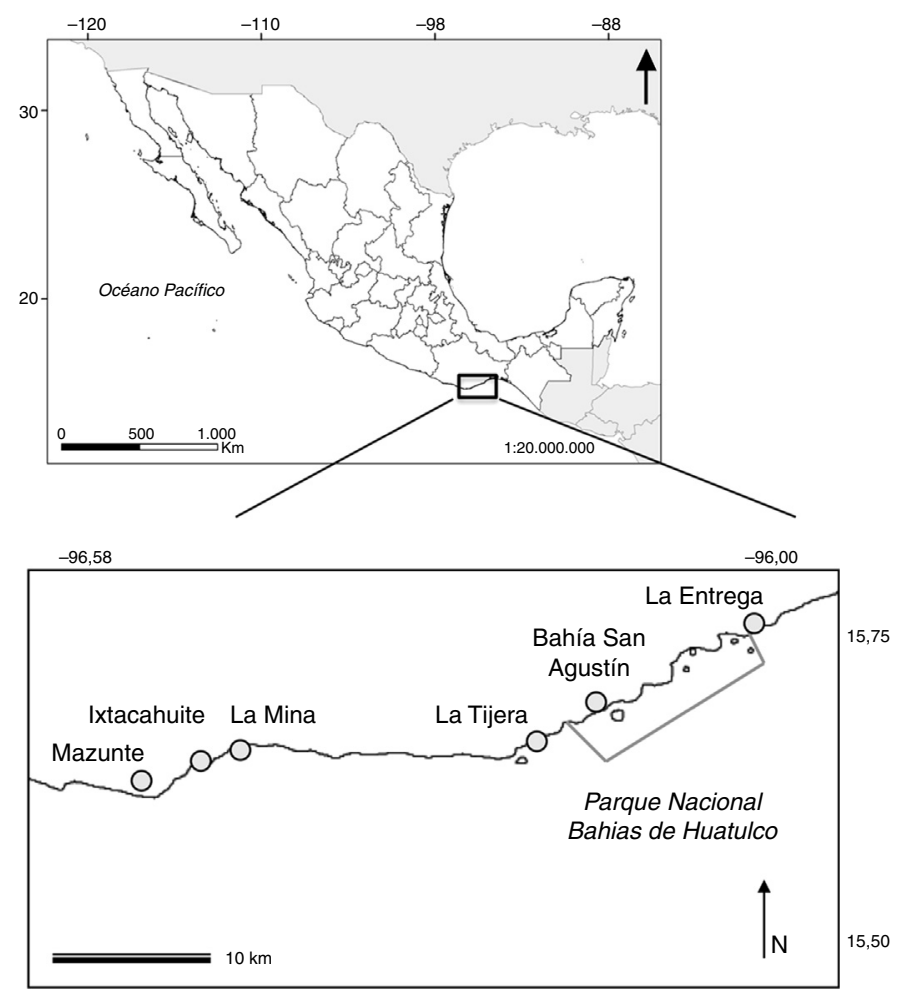

Figura 1. Área de estudio, con los sitios donde se llevaron a cabo los muestreos. men de mareas para el litoral oaxaqueño es mixto semidiurno, con 2 pleamares y 2 bajamares diarias, aunque la fase de marea cambia muy lentamente (Wyrtki, 1965). Finalmente, la profundidad media de la termoclina es de 60 a $80 \mathrm{~m}$, que se incrementa hacia el sur del golfo de Tehuantepec (Fiedler, 1992; Lluch-Cota, Álvarez-Borrego, Santamaría-Del Ángel, Hernández-Vázquez y Müller-Karger, 1997).

Al igual que otras comunidades arrecifales del Pacífico tropical mexicano, los arrecifes de Oaxaca son de franja. Las estructuras están bien consolidadas y se consideran entre las mejor desarrolladas de México; ocupan hasta 34.7 ha (LópezPérez, UAM-Iztapalapa, datos no publicados) en área y hasta $6 \mathrm{~m}$ de espesor (Carriquiry, Cupul-Magaña, Rodríguez-Zaragoza y Medina-Rosas, 2001; Glynn y Leyte-Morales, 1997). El ensamblaje coralino está compuesto básicamente por los géneros Pocillopora, Porites y Pavona (López-Pérez y HernándezBallesteros, 2004); sin embargo, la variedad de especies es baja y el paisaje arrecifal está dominado por Pocillopora spp., que es básicamente responsable de la construcción de los arrecifes. Existe una zonación muy clara en la cual los pocilopóridos habitan en aguas someras - hasta $5 \mathrm{~m}$ - con un incremento gradual de Pavona y Porites en áreas profundas (Carriquiry y ReyesBonilla, 1997; Glynn y Leyte-Morales, 1997; Reyes-Bonilla y Leyte-Morales, 1998).

El trabajo de campo se llevó a cabo durante el mes de abril del 2008 mediante buceo autónomo. Se obtuvieron de 12 a 45 fragmentos de cada una de las 9 especies pertenecientes a 3 géneros de coral: Pavona, Pocillopora y Porites, con el fin de determinar la composición de sus simbiontes. La identificación de las colonias se efectuó a partir de criterios puramente morfológicos, y con base en los caracteres diagnósticos y las imágenes provistas por Cortés-Núñez y Guzmán-Espinal (1998); Hickman (2008); Ketchum y Reyes-Bonilla (2001) y Veron (2000). Las muestras fueron tomadas en 6 localidades y a varios niveles de profundidad, procurando extraer por lo menos un ejemplar de cada género cada $2 \mathrm{~m}$ (de 2 a $15 \mathrm{~m}$ ). Se tomaron pequeños fragmentos de las colonias ( $\sim 2 \mathrm{~cm}^{2}$ de superficie de tejido) con ayuda de un cincel y un martillo. El material se colocó en viales de $1 \mathrm{ml}$ y se preservó en alcohol absoluto. Las muestras fueron transportadas al Laboratorio Mueller, de la Universidad Estatal de Pensilvania, para su análisis genético.

El trabajo de laboratorio consistió en la extracción de ADN de Symbiodinium mediante el protocolo descrito por LaJeunesse et al. (2003), utilizando un kit de purificación genómica Wizard (Promega®). Se utilizó el marcador genético ITS2, empleando cebadores diseñados por LaJeunesse y Trench (2000). Para la amplificación por PCR se utilizaron los siguientes criterios: aproximadamente $1 \mu \mathrm{l}$ de ADN (previamente diluido en $200 \mu \mathrm{l}$ de agua estéril desionizada), se añadió a una mezcla fría con $2.5 \mu \mathrm{l}$ de DNTP, $2.5 \mu \mathrm{l}$ de MgCL, $2.5 \mu \mathrm{l}$ de solución buffer, $18 \mu \mathrm{l}$ de agua estéril desionizada, $0.25 \mu \mathrm{l}$ del cebador A «ITSIntfor» (5'GAATTGCAGA ACTCCGTG-3'); $0.75 \mu \mathrm{l}$ del B «ITS2CLAMP» (5'CGCCCGCCGCGCCCCGCGCC CGTCCCGCCG CCCCCGCCC GGGATCCATATGCTTAAGTT CAGCGGGT-3') (Sheffield, Cox, Lerman y Myers, 1989), $0.13 \mu \mathrm{l}$ de Taq ADN polimerasa recombinante y $18 \mu \mathrm{l}$ de agua desionizada para obtener un volumen total por tubo de 
$25 \mu l$ de reacción. Esta mezcla se incubó en un termociclador a $95^{\circ} \mathrm{C}$ por 3.30 min seguido por 20 ciclos (desnaturalización a $94.0^{\circ} \mathrm{C}$ por $0.45 \mathrm{~s}$; alineamiento a $60.0^{\circ} \mathrm{C}$ por $0.40 \mathrm{~s}$; polimerización a $72.0^{\circ} \mathrm{C}$ por $0.30 \mathrm{~s}$ ), con una disminución de $1{ }^{\circ} \mathrm{C}$ cada ciclo en la temperatura de alineamiento, y otros 15 a 18 ciclos en las mismas condiciones, pero la temperatura de alineamiento se mantuvo constante en $52{ }^{\circ} \mathrm{C}$. El ADN total se evaluó por electroforesis en gel de agarosa al $1 \%$ con una concentración final de bromuro de etidio de $2 \mu \mathrm{l}$, a un voltaje constante de $97 \mathrm{~V}$ durante aproximadamente $10 \mathrm{~min}$.

Bajo luz ultravioleta se seleccionó el material de alto peso molecular, evaluando la estructura de la banda. Para el presente trabajo los productos de reacción fueron cargados en un gel de $8 \%$ de acrilamida (a gradiente desnaturalizante del 45 al $80 \%$, el $100 \%$ consistía en $7 \mathrm{M}$ de urea y $40 \%$ de formamida desionizada). Los productos de PCR fueron colocados en el gel con solución de carga de ficol al 2\% (ficol-400 2\%, $10 \mathrm{mM}$ Tris-HCL, pH 7.8, 1 mM EDTA, $1 \%$ de azul de bromofenol) y separados por electroforesis durante $15 \mathrm{~h}$ a un voltaje constante de $150 \mathrm{~V} \mathrm{y}$ temperatura de $60^{\circ} \mathrm{C}$. Al término de la prueba, el gel fue teñido en una solución de 2-3 $\mu \mathrm{l}$ de bromuro de etidio y $10 \mathrm{ml}$ de buffer TAE 1X, incubándose en oscuridad total durante 25-35 min. El gel se enjuagó con agua desionizada y fue transferido a una caja de luz UV, donde fue observado y fotografiado. Se llevó a cabo una revisión visual de los geles que permitió una identificación de los clados de zooxantela por la posición de la banda más conspicua en el gel, ya que existe un patrón de migración preestablecido para cada clado (LaJeunesse, 2001).

Para el análisis de resultados, se evaluó la similitud cualitativa y cuantitativa entre las diferentes infracomunidades. En el primer caso, se tomaron en cuenta los datos de presencia/ausencia de los genotipos en cada localidad y, para el segundo, las abundancias de cada genotipo a diferentes niveles de profundidad. Se hizo un análisis de bondad de ajuste $\left(x^{2}\right)$ para comparar las frecuencias observadas y esperadas de los clados a partir de la hipótesis nula de una distribución homogénea entre las especies de coral, y otra similar para comparar entre los estratos de profundidad (Daniel, 2002).

Se calculó la distancia genética de Nei (1978) para conocer la similitud entre localidades con respecto a la composición de los tipos de zooxantela, empleando como alelos los genotipos de cada individuo. Adicionalmente, se calculó la distancia geográfica lineal entre las localidades de muestreo con ayuda del programa Google Earth y, por último, se comparó la distan-

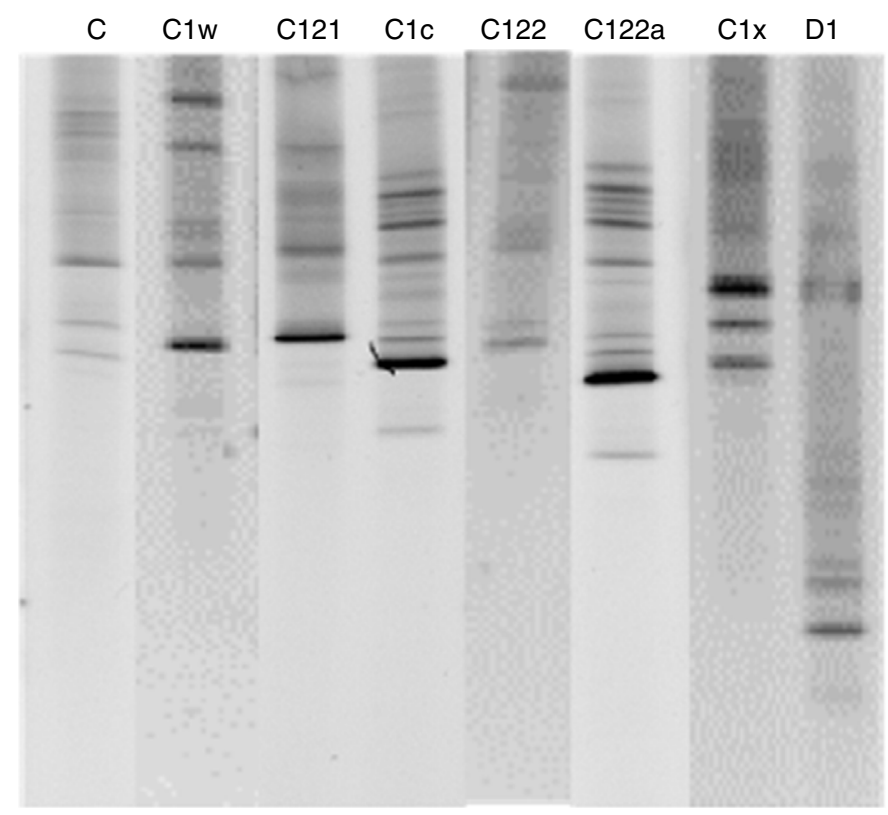

Figura 2. Perfil de los genotipos de dinoflagelados simbióticos diagnosticados por PCR-DGGE para la costa de Oaxaca. La letra mayúscula indica el clado, el número representa el tipo de ITS2 y la letra minúscula denota que existe un parálogo característico de ADN.

cia genética de los clados contra la distancia geográfica entre localidades, con el fin de determinar una posible relación de aislamiento por distancia.

\section{Resultados}

Para el litoral de Oaxaca se encontraron 3 géneros y 9 especies de coral (tabla 1). El género más común presente en todas las localidades y profundidades fue Pocillopora —que, además, fue el más diverso con un total de 6 especies-, seguido por Pavona con 2 especies y finalmente Porites con solamente una. En las 6 localidades muestreadas se registró un total de 8 genotipos distintos pertenecientes a 2 de los principales clados dentro del género Symbiodinium: C y D (fig. 2). La mayor diversidad de Symbiodinium se encontró en Porites (fig. 3), identificando 6 genotipos dentro de este género. De este último, el 51\% de los ejemplares mostraron asociación con el simbionte C1x y el 17\% con el $\mathrm{C} 122$, mientras que todas las especies y ejemplares de Pocillopora presentaron el genotipo D1. Finalmente, el 83\%

Tabla 1

Especies de corales hospederos analizados en el litoral de Oaxaca.

\begin{tabular}{|c|c|c|}
\hline Hospedero analizado & Número de ejemplares recolectados & Profundidad de colecta $(\mathrm{m})$ \\
\hline Pocillopora elegans (Dana, 1846) & 1 & 7 \\
\hline Pocillopora meandrina (Dana, 1846) & 7 & $2.6-9$ \\
\hline Pocillopora verrucosa (Ellis y Solander, 1786) & 13 & $4.2-10.2$ \\
\hline Pocillopora damicornis (Linnaeus, 1758) & 32 & $2.6-12.1$ \\
\hline Pocillopora eydouxi (Milne-Edwards y Haime, 1860) & 15 & $4-9.9$ \\
\hline Pocillopora capitata (Verrill, 1864) & 11 & $3.5-8.8$ \\
\hline Pavona varians (Verrill, 1864) & 6 & $7.5-8.7$ \\
\hline Pavona gigantea (Verrill, 1869) & 30 & $5.2-15.3$ \\
\hline Porites panamensis (Verrill, 1866) & 24 & $5-13.8$ \\
\hline
\end{tabular}


Tabla 2

Tipos de simbionte encontrados en cada localidad.

\begin{tabular}{|c|c|c|c|c|c|c|c|c|c|c|}
\hline & $\mathrm{C}$ & C1w & $\mathrm{C} 121$ & $\mathrm{C} 1 \mathrm{c}$ & $\mathrm{C} 122$ & $\mathrm{C} 122 \mathrm{a}$ & $\mathrm{C} 1 \mathrm{x}$ & D1 & Tamaño de muestra & Número total de simbiontes \\
\hline Mazunte & & & & & & & 2 & 10 & 12 & 2 \\
\hline Ixtacahuite & 1 & & & 5 & & & 5 & 10 & 21 & 4 \\
\hline La Mina & 1 & & & 1 & & & 4 & 11 & 17 & 4 \\
\hline La Tijera & 4 & 1 & & 3 & & & 1 & 12 & 21 & 5 \\
\hline San Agustín & & 1 & 2 & 3 & & & & 17 & 23 & 4 \\
\hline La Entrega & & & & 18 & 4 & 2 & & 21 & 45 & 4 \\
\hline
\end{tabular}
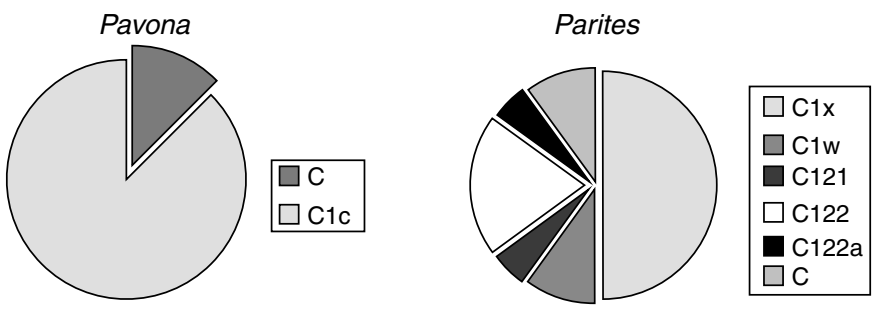

Figura 3. Proporción de la diversidad genotípica de dinoflagelados simbiontes en los géneros Pavona y Porites.

de las colonias del género Pavona estuvieron asociadas con el genotipo C1c y el $17 \%$ con el C (fig. 3). Los resultados indican que el genotipo D1 estuvo presente en todos los sitios debido a su asociación con Pocillopora, mientras que los C122 y C122a se identificaron una sola vez —en La Entrega-, y el C121 solo en San Agustín (tabla 2).

El genotipo $\mathrm{C}$ se presentó en 3 localidades, pero asociado a diferentes hospederos; en Ixtacahuite se observó en Pavona gigantea, en La Mina apareció en Porites panamensis, y en La Tijera fue posible observar este tipo de simbionte en ambas especies. El número de muestras por sitio fue independiente del número de genotipos identificados, sin mostrar una tendencia específica y sin favorecer a las localidades en las que hubo un mayor esfuerzo de colecta. Por ejemplo, en La Entrega se tomaron 45 muestras y se lograron identificar 4 genotipos, mismo número que en La Mina, en donde solo se recolectaron 17 fragmentos de coral.

En cuanto a la composición de simbiontes en las localidades, la mayor diversidad se encontró en La Tijera y la menor en Mazunte (tabla 2). La Mina e Ixtacahuite resultaron idénticas entre sí y muy similares a las de La Tijera, ya que esta se diferencia de las anteriores solamente en la presencia de un genotipo adicional, el $\mathrm{C} 1 \mathrm{w}$. Mazunte estuvo cercanamente relacionada con las anteriores por compartir los genotipos D1 y C1x, que fueron los únicos 2 simbiontes encontrados en esta localidad. Finalmente, las zonas más divergentes fueron San Agustín, que compartió genotipos con las anteriores, pero tiene uno exclusivo (C121), y La Entrega, donde aparecieron 2 genotipos que no se presentaron en ningún otro sitio (C122, C122a; tabla 2).

Por otra parte, la composición de especies de simbiontes fue variable a lo largo del gradiente de profundidad. Los estratos someros (de 2 a $4 \mathrm{~m}$ ) fueron idénticos entre sí y mostraron la menor riqueza, mientras que las profundidades intermedias (de 5 a $9 \mathrm{~m}$ ) fueron similares entre sí y reflejaron la mayor riqueza. Por último, los estratos más profundos fueron los más distintos a los anteriores, e incluso entre sí mismos, y presentaron una diversidad de simbiontes relativamente baja. Estas diferencias estuvieron estrechamente relacionadas con el tipo de coral presente en cada estrato de profundidad. El género Pocillopora, dominado por el clado $\mathrm{D}$, exhibió una propensión por profundidades que van de 4 a $8 \mathrm{~m}$ (tabla 3), y Pavona apareció principalmente de los 6 a los $10 \mathrm{~m}$. La prueba de bondad de ajuste mostró diferencias significativas en las proporciones de los clados por estrato de profundidad en Pocillopora y Pavona $\left(x^{2}=49.29, p<0.001\right.$, g.1. 5; $x^{2}=27.3, p<0.001$, g.l. 5). En contraste, Porites tuvo una distribución más homogénea a lo largo del gradiente de profundidad sin mostrar una preferencia evidente hacia algún estrato, por lo que no se evidenciaron diferencias significativas entre los rangos de profundidad de distribución de este género $\left(x^{2}=6.8, p>0.005\right.$, g.1. 4). La mayor diversidad de Symbiodinium se encontró de los 8 a los $10 \mathrm{~m}$, con un total de 7 genotipos distintos a esta profundidad (tabla 3).

Los simbiontes con la mayor frecuencia de aparición fueron los tipos D1 y C1c, los mismos que se encontraron exclusivamente en Pocillopora y Pavona. El genotipo D1 se encontró en todos los estratos muestreados, pero fue más abundante en áreas someras por la dominancia del género Pocillopora (tabla 3). De acuerdo con el análisis de bondad de ajuste, solamente el simbionte D1 exhibió diferencias significativas en sus frecuencias acumuladas para los 2 estratos de profundidad (somero y profundo; $x^{2}=15.12, p<0.001$, g.l. 1). Para el resto, la prueba no mostró diferencias, aun cuando el C122 se encontró exclusivamente en el estrato profundo, lo cual indica que estos simbiontes poseen amplios intervalos batimétricos, sin tener una preferencia muy marcada hacia algún nivel de profundidad.

Los valores de la distancia genética entre las poblaciones de Symbiodinium se encontraron entre 0.018 y 0.312 (tabla 4). La menor diferenciación correspondió a las poblaciones de Mazunte-La Mina y Mazunte-San Agustín, mientras los valores con mayor distancia genética se dieron entre La Entrega-Mazunte y La Mina-La Entrega. Además, fue posible observar una relación positiva entre la distancia geográfica de las localidades y la distancia genética entre las poblaciones de Symbiodinium.

\section{Discusión}

En comparación con estudios de zooxantelas que se han realizado en distintos lugares del mundo (LaJeunesse, 2002; LaJeunesse et al., 2003, 2004; LaJeunesse et al., 2008), los resultados del presente trabajo indican que la diversidad de Symbiodinium en la costa de Oaxaca es relativamente alta, en 
Tabla 3

Frecuencias observadas de los tipos de simbiontes dinoflagelados a distintas profundidades y su frecuencia de aparición acumulada en 2 estratos: somero (0-7 m) y profundo $(>8 \mathrm{~m})$

\begin{tabular}{|c|c|c|c|c|c|c|c|c|c|}
\hline Profundidades (m) & $\mathrm{C}$ & $\mathrm{C} 1 \mathrm{w}$ & $\mathrm{C} 121$ & $\mathrm{C} 1 \mathrm{c}$ & $\mathrm{C} 122$ & $\mathrm{C} 122 \mathrm{a}$ & $\mathrm{C} 1 \mathrm{x}$ & D1 & Total \\
\hline $2-3.9$ & 0 & 0 & 0 & 0 & 0 & 0 & 0 & 6 & 6 \\
\hline $4-5.9$ & 1 & 1 & 1 & 2 & 0 & 0 & 1 & 25 & 31 \\
\hline $6-7.9$ & 3 & 0 & 0 & 13 & 0 & 1 & 5 & 27 & 49 \\
\hline $8-9.9$ & 0 & 1 & 1 & 10 & 4 & 1 & 1 & 19 & 37 \\
\hline 10-11.9 & 1 & 0 & 0 & 2 & 0 & 0 & 4 & 3 & 10 \\
\hline 12-13.9 & 0 & 0 & 0 & 1 & 0 & 0 & 1 & 1 & 3 \\
\hline $14>$ & 1 & 0 & 0 & 2 & 0 & 0 & 0 & 0 & 3 \\
\hline Total & 6 & 2 & 2 & 30 & 4 & 2 & 12 & 81 & 139 \\
\hline Somero & 4 & 1 & 1 & 15 & 0 & 1 & 6 & 58 & 86 \\
\hline Profundo & 2 & 1 & 1 & 15 & 4 & 1 & 6 & 23 & 53 \\
\hline
\end{tabular}

especial dentro del género Porites, en el cual hubo 6 genotipos diferentes en solamente 24 colonias muestreadas dentro de una franja costera relativamente corta $(<50 \mathrm{~km})$. El género Pavona, con 2 tipos de simbiontes, se comportó de forma similar a las otras 2 regiones del Pacífico mexicano (LaJeunesse et al., 2003), en donde este coral alberga 1 o 2 tipos de alga. Por su parte, Pocillopora, al solo tener clado D en Oaxaca, exhibió una diversidad extremadamente baja, ya que se analizaron más de 70 colonias de 6 especies. El género se ha encontrado asociado a diversos clados de simbiontes en el Pacífico (LaJeunesse et al., 2004; Magalon, Flot y Baudry, 2007).

En Oaxaca, el genotipo más frecuente fue el D1, ya que apareció en todas las colonias muestreadas del género Pocillopora. Esta dominancia se observa también en otros lugares del Pacífico mexicano (LaJeunesse et al., 2008; Paz-García, LaJeunesse, Chávez-Romo, Correa-Sandoval y Reyes-Bonilla, 2008), lo que indica que este genotipo es generalista a distintas profundidades, y común en el oeste de las Américas. Se ha observado que, en periodos de estrés térmico, los corales que albergan simbiontes pertenecientes al clado $\mathrm{C}$ han experimentado blanqueamiento por alta temperatura, mientras que los del clado D se han mantenido sanos (Baker, Starger, McClanahan y Glynn, 2004). Esto lleva a la suposición de que este último clado es más tolerante al estrés ambiental (Baker et al., 2004, Fabricius, Mieog, Colin, Idip y van Oppen, 2004; Mostafavi, Reza, Shahhosseiny, Hoegh-Guldberg y Weng, 2007). Lo anterior ha sido demostrado mediante la presencia del clado D en sitios con fluctuaciones extremas de temperatura, luz y salinidad (Ulstrup y van Oppen, 2003), o bajo la influencia de material terrígeno y alta turbi- dez (Chen, Yang, Wei, Tsai y Fang, 2005; van Oppen, Palastra, Piquet y Miller, 2001). En este sentido, la presencia del clado D en la costa de Oaxaca pudiera estar asociada con características ambientales similares, pues la influencia de las surgencias del golfo de Tehuantepec estacionalmente modifica las condiciones de productividad y temperatura (Lluch-Cota et al., 1997), además de que el aporte de sedimentos por ríos varía notablemente entre la época de secas y de lluvias (Granja-Fernández y López-Pérez, 2008). Otra posible explicación de alta presencia del clado D1 es que las especies del género Pocillopora son hermafroditas (Carpizo-Ituarte et al., 2011; Glynn et al., 1991), además de que se pueden reproducir de manera asexual por fragmentación (Chávez-Romo y Reyes-Bonilla, 2007; López-Pérez, Mora-Pérez y Leyte-Morales, 2007). En estas condiciones, la alta prevalencia de clones podría determinar la dominancia de D1.

Dentro del Pacífico oriental ocurrió un evento de blanqueamiento masivo resultado del fenómeno de calentamiento del agua, provocado por la oscilación sureña de El Niño en 1997/1998. En respuesta a lo anterior, en Panamá se observó que las colonias de Pocillopora que albergaban simbiontes del clado D se volvieron dominantes al aumentar del 26 al $61 \%$ en frecuencia para 2001 (Glynn, Maté, Baker y Calderón, 2001). En México también fueron muy afectadas las comunidades coralinas de Oaxaca, con una mortalidad de más del 60\% (ReyesBonilla et al., 2002), y en la actualidad el 100\% de las colonias de Pocillopora albergan el clado D. A este respecto, Baker et al. (2004) proponen que lo anterior ocurre en corales que se están recuperando de un evento de blanqueamiento. Aunque no se sabe

Tabla 4

Distancia genética entre infrapoblaciones de Symbiodinium en las localidades muestreadas del litoral de Oaxaca y distancia geográfica (km) entre ellas (señalado en negritas).

\begin{tabular}{|c|c|c|c|c|c|c|}
\hline \multicolumn{7}{|c|}{ Distancia geográfica } \\
\hline \multicolumn{7}{|c|}{ Distancia genética } \\
\hline Ixtacahuite & 0.132 & ------------ & 0.71 & 24.70 & 27.06 & 38.89 \\
\hline La Mina & 0.018 & 0.064 & ------------ & 24.22 & 26.56 & 38.34 \\
\hline La Tijera & 0.087 & 0.111 & 0.073 & --------- & 2.34 & 14.88 \\
\hline
\end{tabular}


qué simbiontes se encontraban en las colonias coralinas antes del evento, podría inferirse que lo observado en Oaxaca se debe a la sobrevivencia diferencial de los corales portadores del clado D1, como fue propuesto por LaJeunesse et al. (2010) en todo el Pacífico oriental. La ausencia de recolonización del clado $\mathrm{C}$ en Pocillopora llama la atención, debido a que a nivel mundial el género se asocia principalmente con zooxantelas pertenecientes al clado C (Magalon et al., 2007). Consideramos que, además de la alta mortalidad causada por el evento de 1997-1998, el efecto de filtro biogeográfico que genera la barrera del Pacífico oriental (Glynn y Ault, 2000) ha evitado migraciones de organismos del género Pocillopora que sean portadores del clado $\mathrm{C}$ provenientes de otras regiones.

En los géneros Porites y Pavona se encontraron dinoflagelados simbiontes pertenecientes al clado $\mathrm{C}$, el cual domina los corales del océano Pacífico con más de 100 tipos distintos descritos hasta el 2004 (Pochon, LaJeunesse y Pawlowski, 2004), y es el grupo de simbiontes que contiene el mayor número de variantes a nivel mundial (Knowlton y Rohwer, 2003; LaJeunesse, 2002; LaJeunesse et al., 2003, 2004). En los corales pétreos de Oaxaca, el clado $\mathrm{C}$ se encontró representado por 7 diferentes genotipos, de los cuales 5 se identificaron exclusivamente en Porites, otro se encontró exclusivamente en Pavona y el último se observó en ambos géneros (fig. 3). La presencia del clado C en Oaxaca puede tener una explicación histórica o evolutiva, pues P. panamensis es especie hermana de Porites colonensis Zlatarski 1990 del Atlántico, la cual alberga el clado C1 (Forsman, Hunter, Fox y Wellington, 2006; LaJeunesse, 2002). Asimismo, $P$. gigantea podría descender de especies del género que colonizó el Pacífico oriental durante el Plio-Pleistoceno desde el Pacífico central (López-Pérez y Budd, 2009), en donde el clado $\mathrm{C}$ es actualmente dominante.

Al comparar la diversidad genética de Symbiodinium con la estrategia reproductiva de su hospedero en Oaxaca, fue posible observar una tendencia que indica que la reproducción sexual del coral se asocia con una mayor diversidad de simbiontes, mientras que la asexual se asocia con una menor diversidad de estos. Por ejemplo, la especie $P$. panamensis, en la que se encontró la mayor diversidad de simbiontes, se reproduce de manera sexual y es gonocórica con fertilización interna (Carpizo-Ituarte et al., 2011). Este coral es incubador y tiene transmisión vertical de zooxantelas, por lo que sus larvas plánulas adquieren sus simbiontes directamente de la colonia madre (Glynn et al., 1994). En este modo de transmisión, la selección natural ejercida sobre el hospedero, una vez que sale de la colonia parental y se incorpora a la vida libre, promueve de manera indirecta la diversificación del tipo de simbiontes en las infracomunidades (Douglas, 1998; Loh, Loi, Carter y Hoegh-Guldberg, 2001), y esto podría estar favoreciendo la variabilidad genética de las zooxantelas asociadas a este coral en la costa de Oaxaca. Por otra parte, Pocillopora tiene un solo clado (D1) y su modo de reproducción local es principalmente por fragmentación, ya que López-Pérez et al. (2007) encontraron solo un recluta sexual en un año de muestreo en sustratos artificiales, mientras que Rodríguez-Troncoso, Carpizo-Ituarte, Leyte-Morales, Chi-Barragán y Tapia-Vázquez (2011) mostraron que pocas especies del género en Oaxaca alcanzan la madurez de sus gametos. La reproducción asexual mantiene la homogeneidad genética del holobionte y, por ello, la posibilidad de variación de la zooxantela en el tiempo tiende a ser mínima.

Un tercer caso lo presenta Pavona, que se ha registrado como hermafrodita; se reproduce sexualmente en un periodo de primavera-verano y libera sus gametos a la columna de agua en donde estos son fertilizados (Carpizo-Ituarte et al., 2011; Glynn et al., 1996; Santiago-Valentín et al., 2015). Este género tuvo una diversidad de simbiontes intermedia comparado con Porites y Pocillopora, y al mismo tiempo su estrategia reproductiva representa un punto entre la fragmentación que presenta Pocillopora y la incubación de larvas que lleva a cabo Porites. Finalmente, en el Caribe también es posible observar este patrón de diversidad genética del simbionte con base en las características reproductivas del hospedero, y aquellas especies que son gonocóricas e incuban sus larvas como Porites astreoides Lamarck, 1816 y Porites furcata Lamarck, 1816 poseen más de 2 tipos de simbiontes, mientras que las que tienen fertilización externa o son hermafroditas como Acropora cervicornis Lamarck, 1816 albergan un solo tipo de alga (LaJeunesse, 2002).

La composición de clados de Symbiodinium en las 6 localidades fue diferente, a pesar de que entre estas la distancia no excede los $50 \mathrm{~km}$. Esta diferenciación genética se puede promover por la presencia de barreras que impidan un flujo genético entre localidades, ya sean estas de tipo oceanográfico - dirección y velocidad de las corrientes, gradientes de temperatura o salinidad-, ya sea que eviten el reclutamiento larval —-sedimentación, competencia por el sustrato-, ya sea por actividades humanas - turismo, contaminación-. Respecto a esto último, López-Pérez y Hernández-Ballesteros (2004) observaron que San Agustín ha tenido un menor impacto por perturbaciones naturales que La Entrega, localidad que, además, ha tenido un mayor impacto turístico.

Se ha registrado que la distribución batimétrica de los corales arrecifales puede ser dependiente del tipo de simbionte que albergan. Iglesias-Prieto et al. (2004) evidenciaron que, en el golfo de California, los dinoflagelados del clado D que habitan colonias de Pocillopora verrucosa —especie típica de agua somera-, toleran mayor irradiancia que el clado $\mathrm{C}$ de Pavona gigantea —especie que normalmente se presenta a mayor profundidad-, y esto podría explicar la zonación observada en Oaxaca, en donde el género Pocillopora exhibió una preferencia por profundidades que van de 4 a $8 \mathrm{~m}$, mientras Pavona se encontró de los 6 a los $10 \mathrm{~m}$. Cabe indicar también que Porites tuvo una distribución más homogénea a lo largo de la profundidad, sin mostrar preferencia por algún estrato, quizá por su mayor variedad de clados que le permitan una mayor tolerancia a los cambios de iluminación.

En relación con el flujo genético, los resultados muestran claramente que la distancia geográfica influye negativamente sobre la conectividad, ya que se observó que Ixtacahuite y La Mina tuvieron una composición de clados idéntica y son las localidades más cercanas, mientras Mazunte y La Entrega son las localidades más distantes y menos similares. En el caso de los corales del Pacífico mexicano, Chávez-Romo et al. (2009) observaron la misma relación para las poblaciones de Pocillopora damicornis, Paz-García et al. (2008) las documentaron 
para Porites panamensis en el golfo de California y SaavedraSotelo et al. (2011) para Pavona gigantea. En la región de Oaxaca, cuando la distancia entre poblaciones de Symbiodinium es mayor, el intercambio genético se vuelve muy limitado. Considerando la región completa del Pacífico mexicano, podría inferirse que existe una clara estructura y distintos demes del mismo tipo de zooxantela, y posiblemente exista baja conectividad entre poblaciones de Symbiodinium de Oaxaca y del noroeste de México.

Recientemente, Fabina et al. (2012) han reconocido que los altos niveles de diversidad encontrados en corales y en Symbiodinium resultan en un número potencialmente alto de combinaciones coral-simbionte con características funcionales únicas. En el caso del presente estudio y como se indica en la metodología, las especies de corales de la costa de Oaxaca fueron reconocidas siguiendo criterios morfológicos, por lo que las combinaciones coral-simbionte que se lograron reconocer están basadas en la forma de la colonia y el tipo de clado. Estudios futuros respecto a la identidad genética de las especies de corales de la costa de Oaxaca permitirán determinar la diversidad de los holobiontes presentes en la región. Finalmente, el presente estudio es el primero en documentar la asociación de Porites panamensis con los clados C121, C122, C122a, C1w y C1x, lo que indica un cierto nivel de aislamiento de esta especie en la región. Este fenómeno puede explicarse porque aparentemente la especie tiene poco tiempo larval de vida libre y sus larvas se asientan a pocos metros de las colonias madre (Glynn y Ault, 2000; Reyes-Bonilla y Calderón-Aguilera, 1994).

\section{Agradecimientos}

Se agradece a la Universidad Estatal de Pensilvania y a la Universidad del Mar por el apoyo dado para realizar este trabajo. Asimismo, se agradece a la National Science Foundation (IOB 544854 a TLJ y IOB 544765 a M. Warner) y al Conacyt (80228 a RALP) por la aportación de los fondos necesarios para efectuar la investigación.

\section{Referencias}

Baker, A. C. (2003). Flexibility and specificity in coral-algal symbiosis: diversity, ecology, and biogeography of Symbiodinium. Annual Review of Ecology and Systematics, 34, 661-689.

Baker, A. C., Glynn, P. W. y Riegl, B. (2008). Climate change and coral reef bleaching: an ecological assessment of long-term impacts, recovery trends and future outlook. Estuarine, Coastal and Shelf Science, 80, 435-471.

Baker, A. C., Starger, C. J., McClanahan, T. R. y Glynn, P. W. (2004). Corals' adaptive response to climate change. Nature, 430, 741

Carpizo-Ituarte, E., Vizcaíno-Ochoa, V., Chi-Barragán, G., Tapia-Vázquez, O., Cupul-Magaña, A. L. y Medina-Rosas, P. (2011). Evidencia de reproducción sexual en los corales hermatípicos Pocillopora damicornis, Porites panamensis y Pavona gigantea en bahía de Banderas, Pacífico mexicano. Ciencias Marinas, 37, 97-112.

Carriquiry, J. D. y Reyes-Bonilla, H. (1997). Estructura de la comunidad y distribución geográfica de los arrecifes coralinos de Nayarit, Pacífico de México. Ciencias Marinas, 23, 227-248.

Carriquiry, J. D., Cupul-Magaña, A. L., Rodríguez-Zaragoza, F. A. y MedinaRosas, P. (2001). Coral bleaching and mortality in the Mexican Pacific during the 1997-98 El Niño and prediction from a remote sensing approach. Bulletin of Marine Science, 69, 237-249.
Chávez-Romo, H. E. y Reyes-Bonilla, H. (2007). Reproducción sexual del coral Pocillopora damicornis al sur del golfo de California, México. Ciencias Marinas, 33, 495-501.

Chávez-Romo, H. E., Correa-Sandoval, F., Paz-García, D. A., Reyes-Bonilla, H., López-Pérez, R. A., Medina-Rosas, P., et al. (2009). Genetic structure of the scleractinian coral, Pocillopora damicornis, from the Mexican Pacific. Proceedings of the 11th International Coral Reef Symposium, 1, 429-433.

Chen, C. A., Yang, Y. W., Wei, N. V., Tsai, W. S. y Fang, L. S. (2005). Symbiont diversity in scleractinian corals from tropical reefs and subtropical non-reef communities in Taiwan. Coral Reefs, 24, 11-22.

Conanp (Comisión Nacional de Áreas Naturales Protegidas). (2003). Programa de Manejo Parque Nacional de Huatulco. México, D.F: Comisión Nacional de Áreas Naturales Protegidas-Secretaría de Medio Ambiente y Recursos Naturales.

Cortés-Núñez, J. y Guzmán-Espinal, H. M. (1998). Organismos de los arrecifes coralinos de Costa Rica: descripción, distribución geográfica e historia natural de los corales zooxantelados (Anthozoa: Scleractinia) del Pacífico. Revista de Biología Tropical, 46, 55-92.

Daniel, W. D. (2002). Bioestadística: base para el análisis de las ciencias de la salud (Cuarta edición). México, D.F.: Limusa Wiley.

Done, T. J., Ogden, J. C., Wiebe, W. J. y Rosen, B. R. (1996). Biodiversity and ecosystem function of coral reefs. In Functional roles of biodiversity: a global perspective. Chichester, Nueva York: John Wiley y Sons, EUA.

Douglas, A. E. (1998). Host benefit and the evolution of specialization in symbiosis. Heredity, 81, 599-603.

Fabina, N. S., Putnam, H. M., Franklin, E. C., Stat, M. y Gates, R. D. (2012) Transmission mode predicts specificity and interaction patterns in coralsymbiodinium networks. PLoS ONE, 7, e44970. http://dx.doi.org/10.1371/ journal.pone.0044970

Fabricius, K. E., Mieog, J. C., Colin, P. L., Idip, D. y Van Oppen, M. J. H. (2004). Identity and diversity of coral endosymbionts (zooxanthellae) from three Paulan reefs with contrasting bleaching, temperature and shading histories. Molecular Ecology, 13, 2445-2458.

Färber-Lorda, J., Lavín, M. F. y Guerrero-Ruiz, M. A. (2004). Effects of wind forcing on the trophic conditions, zooplankton biomass and krill biochemical composition in the Gulf of Tehuantepec. Deep-Sea Research II, 51, 601-614.

Fiedler, P. C. (1992). Seasonal climatologies and variability of eastern tropical Pacific surface waters. NOAA Technical Report, 109, 1-70.

Forsman, Z., Hunter, C. L., Fox, G. E. y Wellington, G. M. (2006). Is the ITS region the solution to the 'species problem' in corals? Intragenomic variation and alignment permutation in Porites, Siderastrea and outgroup taxa. Proceedings of the 10th International Coral Reef Symposium, 1, 14-23.

García, E. R., Vidal-Zepeda, A. y Hernández, M. G. (1990). Carta de climas. IV.4.10, B. Atlas de México. México, D.F.: Instituto de Geografía, Universidad Nacional Autónoma de México.

Glynn, P. W. (1996). Coral reef bleaching: facts, hypotheses and implications. Global Change Biology, 2, 495-509.

Glynn, P. W. y Ault, J. S. (2000). A biogeographic analysis and review of the far eastern Pacific coral reef region. Coral Reefs, 19, 1-23.

Glynn, P. W. y Leyte-Morales, G. E. (1997). Coral reefs of Huatulco, west Mexico. Reef development in upwelling Gulf of Tehuantepec. Revista de Biología Tropical, 45, 1033-1047.

Glynn, P. W., Colley, S. B., Eakin, C. M., Smith, D. B., Cortés, J., Gassman, N. J., et al. (1994). Reef coral reproduction in the eastern Pacific: Costa Rica, Panama, and Galapagos Islands (Ecuador). II. Poritidae. Marine Biology, 118, 191-208.

Glynn, P. W., Colley, S. B., Gassman, N. J., Black, K., Cortés, J. y Maté, J. L. (1996). Reef coral reproduction in the eastern Pacific: Costa Rica, Panama, and Galapagos Islands (Ecuador). III. Agariciidae (Pavona gigantea and Gardineroseris planulata). Marine Biology, 125, 579-601.

Glynn, P. W., Gassman, N. J., Eakin, C. M., Cortés, J., Smith, D. B. y Guzmán, H. M. (1991). Reef coral reproduction in the eastern Pacific: Costa Rica, Panama, and Galapagos Islands (Ecuador). Marine Biology, 109, $355-368$

Glynn, P. W., Maté, J. L., Baker, A. C. y Calderón, M. O. (2001). Coral bleaching and mortality in Panama and Ecuador during the 1997-1998 El Niño-southern oscillation event: Spatial/temporal patterns and comparisons with the 1982-1983 event. Bulletin of Marine Science, 69, 79-109. 
Granja-Fernández, R. y López-Pérez, R. A. (2008). Sedimentación en comunidades arrecifales de bahías de Huatulco, Oaxaca, México. Revista de Biología Tropical, 56, 1179-1187.

Hickman, C. P. (2008). A field guide to corals and other radiates of Galapagos. Lexington: Sugar Spring Press.

Hoegh-Guldberg, O., Mumby, P. J., Hooten, A. J., Steneck, R. S., Greenfield, P., Gómez, E., et al. (2007). Coral reefs under rapid climate change and ocean acidification. Science, 318, 1737-1742.

Iglesias-Prieto, R. y Trench, R. K. (1997). Photoadaptation, photoacclimation and niche diversification in invertebrate-dinoflagellate symbioses. Proceedings of the 8th International Coral Reef Symposium, 2, 1319-1324.

Iglesias-Prieto, R., Beltrán, V. H., LaJeunesse, T. C., Reyes-Bonilla, H. y Thomé, P. E. (2004). Different algal symbionts explain the vertical distribution of dominant reef corals in the eastern Pacific. Proceedings of the Royal Society of London series B, 271, 1757-1763.

Kessler, W. S. (2006). The circulation of the eastern tropical Pacific: a review. Progress in Oceanography, 69, 181-217.

Ketchum, J. T. y Reyes-Bonilla, H. (2001). Taxonomía y distribución de los corales hermatípicos (Scleractinia) del archipiélago de Revillagigedo, México. Revista de Biología Tropical, 49, 803-848.

Knowlton, N. y Rohwer, F. (2003). Multispecies microbial mutualisms on coral reefs: the host as a habitat. American Naturalist, 162, 52-62.

LaJeunesse, T. C. (2001). Investigating the biodiversity, ecology, and phylogeny of endosymbiotic dinoflagellates in the genus Symbiodinium using the internal transcribed spacer region: In search of a «species» level marker. Journal of Phycology, 37, 866-880.

LaJeunesse, T. C. (2002). Diversity and community structure of symbiotic dinoflagellates from Caribbean coral reefs. Marine Biology, 141, 387-400.

LaJeunesse, T. C. y Trench, R. K. (2000). Biogeography of two species of Symbiodinium (Freudenthal) inhabiting the intertidal sea anemone Anthopleura elegantissima (Brandt). Biological Bulletin, 199, 126-134.

LaJeunesse, T. C., Loh, W. K. W., van Woesik, R., Hoegh-Guldberg, O., Schmidt, G. W. y Fitt, W. K. (2003). Low symbiont diversity in southern Great Barrier Reef corals relative to those of the Caribbean. Limnology and Oceanography, 48, 2046-2054.

LaJeunesse, T. C., Reyes-Bonilla, H. y Warner, M.E. (2007). Spring «bleaching» among Pocillopora in the Sea of Cortez, Eastern Pacific. Coral Reefs, 26, 265-270.

LaJeunesse, T. C., Reyes-Bonilla, H., Warner, M. E., Wills, M., Schmidt, G. W. y Fitt, W. K. (2008). Specificity and stability in high latitude eastern Pacific coral-algal symbioses. Limnology and Oceanography, 53, 719-727.

LaJeunesse, T. C., Smith, R., Walther, M., Pettay, D. T., McGinley, M., Aschaffenburg, M., et al. (2010). Host-symbiont recombination versus natural selection in the response of coral-dinoflagellate symbioses to environmental disturbance. Proceedings of the Royal Society of London series B, 277, 2925-2934.

LaJeunesse, T. C., Thornhill, D. J., Cox, E., Stanton, F., Fitt, W. K. y Schmidt, G. W. (2004). High diversity and host specificity observed among symbiotic dinoflagellates in reef coral communities from Hawaii. Coral Reefs, 23, 596-603.

Lluch-Cota, S. E., Álvarez-Borrego, S., Santamaría-Del Ángel, E. M., Hernández-Vázquez，S. y Müller-Karger，F. E. (1997). El golfo de Tehuantepec y áreas adyacentes: variación espaciotemporal de pigmentos fotosintéticos derivados de satélite. Ciencias Marinas, 23, 329-340.

Loh, W. K., Loi, T., Carter, D. y Hoegh-Guldberg, O. (2001). Genetic variability of the symbiotic dinoflagellates from the wide ranging coral species Seriatopora hystrix and Acropora longicyathus in the Indo-West Pacific. Marine Ecology Progress Series, 222, 97-107.

López-Pérez, R. A. y Budd, A. F. (2009). Coral diversification in the Gulf of California during the Late Miocene to Pleistocene. En M. E. Johnson y J. Ledesma-Vázquez (Eds.), Atlas of coastal ecosystems in the western Gulf of California (pp. 58-71). Tucson, Arizona: The University of Arizona Press.

López-Pérez, R. A. y Hernández-Ballesteros, L. M. (2004). Coral community structure and dynamics in the Huatulco area, western Mexico. Bulletin of Marine Science, 75, 453-472.

López-Pérez, R. A., Benítez-Villalobos, F., López-Ortiz, A. M., López PérezMaldonado, I., Granja-Fernández, M. R. y Domínguez-Gómez, M. T. (2008).
La comunidad arrecifal en isla Cacaluta. En J. M. Domínguez-Licona (Ed.), Diagnóstico de los recursos naturales de la bahía y micro-cuenca de Cacaluta (pp. 243-255). Puerto Ángel, Oaxaca: Universidad del Mar.

López-Pérez, R. A., Mora-Pérez, M. E. y Leyte-Morales, G. E. (2007). Coral (Anthozoa: Scleractinia) recruitment at Bahías de Huatulco, western Mexico: implications for coral community structure and dynamics. Pacific Science, 61, 355-369.

Magalon, H., Flot, J. F. y Baudry, E. (2007). Molecular identification of symbiotic dinoflagellates in Pacific corals in the genus Pocillopora. Coral Reefs, 26, 551-558.

Mostafavi, P. G., Reza, S. M., Shahhosseiny, M. H., Hoegh-Guldberg, O. y Weng, W. K. (2007). Predominance of clade D Symbiodinium in shallowwater reef-building corals of Kish and Larak Islands (Persian Gulf, Iran). Marine Biology, 153, 25-34.

Muller-Parker, G. y D'Elia, C. F. (1997). Interactions between corals and their symbiotic algae. En C. Birkeland (Ed.), Life and death of coral reefs (pp. 96-113). Berlín: Elsevier.

Nei, M. (1978). Estimation of average heterozygosity and genetic distance from a small number of individuals. Genetics, 89, 583-590.

Paz-García, D. A., LaJeunesse, T. C., Chávez-Romo, H. E., Correa-Sandoval, F. y Reyes-Bonilla, H. (2008). Differences in the distribution of Symbiodinium spp. among morphotypes and genotypes of Porites panamensis from the Gulf of California, Mexico. Proceedings of the 11th International Coral Reef Symposium, 1, 164-167.

Pennington, J. T., Mahoney, K. L., Kuwahara, S. V., Kolber, D. D., Calienes, R. y Chávez, F. P. (2006). Primary production in the eastern tropical Pacific: a review. Progress in Oceanography, 69, 285-317.

Peterson, C. H. y Lubchenco, J. (1997). On the value of marine ecosystems to society. En G. C. Daily (Ed.), Nature's Services. Societal dependence on natural ecosystems (pp. 177-194). New York, New York: Island Press.

Pochon, X., LaJeunesse, T. C. y Pawlowski, J. (2004). Biogeographic partitioning and host specialization among foraminiferan dinoflagellate symbionts (Symbiodinium; Dinophyta). Marine Biology, 146, 17-27.

Pochon, X., Montoya-Burgos, J. I., Stadelmann, B. y Pawlowski, J. (2006). Molecular phylogeny, evolutionary rates and divergence timing of the symbiotic dinoflagellate genus Symbiodinium. Molecular Phylogenetics and Evolution, 38, 20-30.

Reyes-Bonilla, H. y Calderón-Aguilera, L. E. (1994). Parámetros poblacionales de Porites panamensis (Anthozoa: Scleractinia) en el arrecife de Cabo Pulmo, México. Revista de Biología Tropical, 42, 121-128.

Reyes-Bonilla, H. y Leyte-Morales, G. E. (1998). Corals and coral reefs of the Puerto Ángel region, Oaxaca, Pacific coast of Mexico. Revista de Biología Tropical, 46, 679-681.

Reyes-Bonilla, H. y López-Pérez, R. A. (1998). Biogeografía de los corales pétreos (Scleractinia) del Pacífico de México. Ciencias Marinas, 24, 211-224.

Reyes-Bonilla, H., Carriquiry, J. D., Leyte-Morales, G. E. y Cupul-Magaña, A. L. (2002). Effects of the El Niño-southern oscillation and the anti-El Niño event (1997-1999) on coral reefs of the western coast of Mexico. Coral Reefs, 21, 368-372.

Rodríguez-Troncoso, A. P., Carpizo-Ituarte, E., Leyte-Morales, G. E., ChiBarragán, G. y Tapia-Vázquez, O. (2011). Sexual reproduction of three coral species from the Mexican south Pacific. Marine Biology, 158, 2673-2683.

Rowan, R. y Powers, D. A. (1991). Molecular genetic identification of symbiotic dinoflagellates (zooxanthellae). Marine Ecology Progress Series, 71, 65-73.

Rowan, R., Knowlton, N., Baker, A. C. y Jara, J. (1997). Landscape ecology of algal symbionts creates variation in episodes of coral bleaching. Nature, $388,265-269$

Saavedra-Sotelo, N. C., Calderón-Aguilera, L. E., Reyes-Bonilla, H., LópezPérez, R. A., Medina-Rosas, P. y Rocha-Olivares, A. (2011). Limited genetic connectivity of Pavona gigantea in the Mexican Pacific. Coral Reefs, 30, 677-686.

Santiago-Valentín, J. D., Rodríguez-Troncoso, A. P., Carpizo-Ituarte, E., Benítez-Villalobos, F., Torres-Hernández, P. y López-Pérez, A. (2015). Reproductive pattern of the reef-building coral Pavona gigantea (Scleractinia: Agariciidae) off southwestern Mexico. Ciencias Marinas, 41, 233-246.

Sheffield, V. C., Cox, D. R., Lerman, L. S. y Myers, R. M. (1989). Attachment of a 40-base-pair G 1 C-rich sequence (GC-clamp) to genomic DNA fragments 
by the polymerase chain reaction results in improved detection of singlebase changes. Proceedings of the National Academy of Science USA, 86, 232-236.

Sheppard, C. R. C., Davy, S. K. y Pilling, G. (2009). The biology of coral reefs. Oxford, Massachusetts: Oxford University Press.

Stanley, G. D. (2003). The evolution of modern corals and their early history. Earth-Science Reviews, 60, 195-225.

Ulstrup, K. E. y van Oppen, M. J. H. (2003). Geographic and habitat partitioning of genetically distinct zooxanthellae (Symbiodinium) in Acropora corals on the Great Barrier Reef. Molecular Ecology, 12, 3477-3484.

Van Oppen, M. J. H. (2007). Perspective: hidden diversity in coral endosymbionts unveiled. Molecular Ecology, 16, 1125-1126.

Van Oppen, M. J. H. y Lough, J. M. (2009). Coral bleaching: patterns, processes, causes and consequences. Berlín: Springer-Verlag.

Van Oppen, M. J. H., Palastra, F. P., Piquet, A. M. T. y Miller, D. J. (2001). Patterns of coral-dinoflagellate associations in Acropora: significance of local availability and physiology of Symbiodinium strains and host-symbiont selectivity. Proceedings of the Royal Society of London series B, 268, 1759-1767.

Veron, J. E. N. (2000). Corals of the World. pp. 1-3. Townsville: Australian Institute of Marine Science, Townsville.

Veron, J. E. N., Hoegh-Guldberg, O., Lenton, T. M., Lough, J. M., Obura, D. O., Pearce-Kelly, P., et al. (2009). The coral reef crisis: the critical importance of $<350 \mathrm{ppm} \mathrm{CO}_{2}$. Marine Pollution Bulletin, 58, $1428-1436$.

Warner, M. E., Fitt, W. K. y Schmidt, G. W. (1996). The effects of elevated temperature on the photosynthetic efficiency of zooxanthellae in hospite from four different species of reef coral: a novel approach. Plant Cell Environment, 19, 291-299.

Wyrtki, K. (1965). Surface currents of the eastern tropical Pacific Ocean. InterAmerican Tropical Tuna Commission Bulletin, 9, 271-304. 